\title{
A Simple Method \\ for Profiling Surface Temperature Distributions by Laser-Ultrasound*
}

\author{
Akira KOSUGI** and Ikuo IHARA*** \\ ** Graduate School of Nagaoka University of Technology \\ ***Department of Mechanical Engineering, Nagaoka University of Technology \\ 1603-1 Kamitomioka, Nagaoka, Niigata 940-2188, Japan \\ E-mail: ihara@mech.nagaokaut.ac.jp
}

\begin{abstract}
In the fields of materials science and engineering, it is often required to measure surface temperatures of heated materials. Although infrared radiations are used for measuring surface temperature, they are not always acceptable for some applications because of the limitation in their ability. Therefore, it is required to develop an alternative technique for surface temperature measurements. In this work, a simple ultrasound method for measuring surface temperature distributions of a heated material is presented. A laser ultrasound scanning technique has been employed for non-contact measurements of surface temperature distributions. The principle of the methods is based on the temperature dependence of a surface acoustic wave (SAW). SAWs are generated at different positions on a material surface consecutively by pulsed laser irradiation scanning (Nd:YAG, wavelength $=1064 \mathrm{~nm}$, energy $200 \mathrm{~mJ} /$ pulse, pulse width $3 \mathrm{~ns}$ ) using a one-dimensional galvanometer scanner, and each SAW is detected at a certain position using a laser interferometer based on photorefractive two-wave mixing (Nd:YAG, wavelength=532 nm, energy $200 \mathrm{~mW}$ ). Based on the laser ultrasound scanning, a number of surfaced temperatures at different positions in series are obtained and are combined together to construct the overall temperature distribution. The proposed ultrasonic method has been applied to the surface temperature measurement of an aluminum plate whose single side is being heated. The surface temperature distributions determined by the present method almost agree with those measured using an infrared camera.
\end{abstract}

Key words: Ultrasonic Measurement, Surface Temperature, Laser-Ultrasound, Simplified Method, Non-Contact Method, Surface Acoustic Wave

\section{Introduction}

Measuring temperature has become one of the fundamental and important issues in the fields of materials science and engineering. In particular, in development and manufacturing of materials, it is strongly required to monitor the temperature distribution and its transient variation of the material being processed at high temperatures because such temperature in the material crucially influences the quality and productivity of final products. In-situ or in-process monitoring technique for the temperature distribution could be beneficial not only for basic research of materials but also for developing an effective process control in materials manufacturing. It is noted that either or both of internal and surface temperatures should be evaluated because surface temperature often reflects the state of internal 
temperature and sometimes they may be independent of each other.

For surface temperature measurements, an infrared radiation technique is well known and widely used. Since this method enables noncontact and real-time measurements of surface temperature distributions, it is convenient for on-line monitoring of heated materials. In this method, however, accurate temperature measurements are often hindered by the different emissivity and reflection of infrared radiation from other heat sources. Such effects due to the emissivity and reflectivity result in the deterioration of measurement accuracy. Thus, the infrared radiation method is not always acceptable for some practical applications, and therefore, development of an alternative method is required.

Ultrasound, because of its high sensitivity to temperature, is expected to be an alternative means for temperature measurements. Because of advantages of ultrasonic measurements such as non-invasive and faster time response, several works on the applications of ultrasound to temperature measurements have been made extensively [1]-[6]. In our previous works [7][8], ultrasonic pulse echo methods for measuring internal temperature distributions were developed. In the method, temperature distributions inside heated materials were successfully determined by an effective ultrasonic method coupled with a one-dimensional finite difference calculation [8]. Recently, the ultrasonic method is modified to be adapted to the determination of surface temperature [9]. In the modification, surface acoustic wave (SAW) which propagates along the surface of material is effectively used for determining surface temperatures. In this method, SAWs on heated materials are measured using a laser ultrasonic technique [10] which provides non-contact measurements of ultrasounds as well as SAWs. Thus, the former method with SAW enables us to perform non-contact measurements of one-dimensional surface temperature distributions of heated materials [9]. It is noted in the method that an inverse analysis coupled with finite difference calculation is employed for the temperature determinations. The inverse analysis to determine temperature distributions in the method [9] is, however, a tedious and time-consuming because of the use of a finite difference calculation. In addition, the former method absolutely requires an appropriate heating model which is often difficult to prepare for practical applications. Therefore, it is beneficial to develop alternative method that overcomes such disadvantages of the former method [9].

In this work, a simple ultrasound method for measuring surface temperature distributions of heated materials is presented. A laser ultrasound scanning technique has been employed for making non-contact measurements of surface temperature distributions. The advantage of the method is that the inverse analysis which is used in the former method [9] is not necessary for obtaining temperature distributions. Therefore, there is no need for preparing a heating model. The proposed method has been applied to the surface temperature measurement of an aluminum plate whose single side is being heated, in order to demonstrate the practicability of the method. A laser interferometer based on photorefractive two-wave mixing [10] is used for SAW measurements of the heated plate.

\section{Method}

It is known that the velocity of ultrasonic wave propagating through a material changes with temperature of the material. The principle of temperature determination by ultrasound is based on the temperature dependence of the ultrasonic wave velocity. In this work, SAWs which propagate along a material surface are employed to estimate surface temperatures on the material surface.

We consider a heated material whose surface has a temperature distribution as shown in Fig. 1. Although the temperature distribution may be a complicated and significant depending on heating condition, the temperature gradient between two points such as $\mathrm{E}_{1}$ and $\mathrm{E}_{2}$ can be assumed to be relatively small and almost linear variation if the distance between 
the two points is short. Surface temperature at such short distance can then easily be determined by the following procedure. We consider here that two waves, SAW $E_{1}$ and SAW $E_{2}$, are generated at two positions, $E_{1}$ and $E_{2}$, respectively and received at position $D$, as shown in Fig. 1(b). The transit time $t$ of SAW between $\mathrm{E}_{1}$ and $\mathrm{E}_{2}$ can then be determined from

$$
t=t_{\mathrm{E}_{1}}-t_{\mathrm{E}_{2}}
$$

where, $t_{\mathrm{E} 1}$ and $t_{\mathrm{E} 2}$ are the transit times of SAW $\mathrm{E}_{1}$ from $\mathrm{E}_{1}$ to $\mathrm{D}$ and $\mathrm{SAW} \mathrm{E}_{2}$ from $\mathrm{E}_{2}$ to $\mathrm{D}$, respectively. Therefore, the velocity of the $\mathrm{SAW}$ propagating from $\mathrm{E}_{1}$ to $\mathrm{E}_{2}$, can simply be determined from

$$
v=\frac{\mathrm{L}_{1}}{t}=\frac{\mathrm{L}_{1}}{t_{\mathrm{E}_{1}}-t_{\mathrm{E}_{2}}}
$$

where, $\mathrm{L}_{1}$ is the distance between $\mathrm{E}_{1}$ and $\mathrm{E}_{2}$. It is noted here that a precise value of the distance $L$ should be known in advance. Fortunately, such value can precisely be determined from the SAW velocity which is calculated from the material property and the transit time $t$ at room temperature. Assuming that the SAW velocity linearly changes with temperature in a certain temperature range, the temperature dependence can be expressed as

$$
v=a T+b
$$

where, coefficients $a$ and $b$ are constants depending on material. It should be noted here that the validity of this assumption of the linearity of temperature dependence of the SAW velocity was verified for steel and aluminum experimentally when the temperature range is between 20 and $200{ }^{\circ} \mathrm{C}$ [8][9]. We now consider that the material is being heated so that surface temperature distribution appears on the material surface and varies with the elapsed time after heating is started. When the temperature gradient between $E_{1}$ and $E_{2}$ can approximately be considered as linear, the average temperature between $E_{1}$ and $E_{2}$ can be obtained from

$$
T_{\mathrm{E}_{1}-\mathrm{E}_{2}}=\left(\frac{\mathrm{L}_{1}}{t_{\mathrm{E}_{1}}-t_{\mathrm{E}_{2}}}-b\right) / a
$$

This temperature $T_{\mathrm{E} 1-\mathrm{E} 2}$ is also the temperature at the middle point between $\mathrm{E}_{1}$ and $\mathrm{E}_{2}$. Since the SAW generation points $\mathrm{E}_{1}$ and $\mathrm{E}_{2}$ and detection point $\mathrm{D}$ are precisely positioned by laser irradiation and they are not influenced by thermal expansion of heated material, the distance

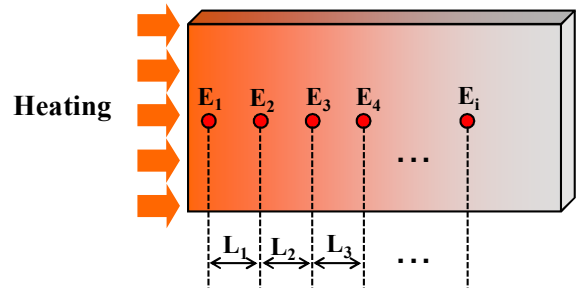

(a)

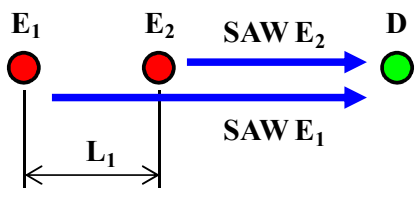

(b)

Fig. 1 (a) Schematic of a heated material having a temperature distribution on the surface, (b) SAWs generation and detection points on the material surface. 
$\mathrm{L}_{1}$ is remaining stable during heating of the material. Therefore, the temperature $\mathrm{T}_{\mathrm{E} 1 \text {-E2 }}$ can easily be determined from Eq. (4) while the transit time of SAW between $E_{1}$ and $E_{2}$ is properly measured.

In the proposed method, an appropriate value of the distance between $E_{1}$ and $E_{2}$ is required to be chosen properly so that the temperature gradient between the two points is relatively small and almost linear. In this work, the SAW generation points can accurately and easily be adjusted by scanning a pulsed laser using a galvanometer scanner so that a number of SAWs in series can be generated at arbitrary position such as $E_{3}, E_{4}$ and $E_{i}$ as shown in Fig. 1. Once SAWs measurements are performed for a number of short distances $\mathrm{L}_{1}, \mathrm{~L}_{2}, \mathrm{~L}_{3}$, and $\mathrm{L}_{\mathrm{i}}$, surface temperature at the middle point of each distance is determined by the procedure mentioned above. The surface temperatures for $\mathrm{L}_{1}, \mathrm{~L}_{2}$, and $\mathrm{L}_{\mathrm{i}}$ are then combined together to construct the surface temperature distribution of the overall distance from $\mathrm{E}_{1}$ to $\mathrm{E}_{\mathrm{i}}$.

\section{Experiment and Result}

In order to verify the practicability of the proposed method, an aluminum plate whose single side is being heated is investigated. An aluminum plate of $30 \mathrm{~mm}$ thickness is used as the specimen. Figure 2 (a) shows the schematic of the experimental setup used. This experimental setup provides a series of noncontact measurements of SAWs on the heated aluminum using a laser-ultrasonic system. The sampling rate of ultrasonic signal is 100 $\mathrm{MHz}$ which is high enough to make precise evaluation of the SAW signal. Figure 2 (b) shows the detail of SAW generation and detection points. SAWs are generated at positions from $E_{1}$ to $E_{6}$ consecutively by pulsed laser scanning irradiation (Nd:YAG, $\lambda=1064 \mathrm{~nm}$, energy $200 \mathrm{~mJ} /$ pulse, pulse width $3 \mathrm{~ns}$ ) using a one-dimensional galvanometer scanner, and each SAW is detected at position D using a laser interferometer based on photorefractive two-wave mixing ( $\mathrm{Nd}$ :YAG, $\lambda=532 \mathrm{~nm}$, energy $200 \mathrm{~mW}$ ). Using the laser-ultrasonic system, surface temperature distributions between $E_{1}$ and $E_{6}$ are determined as a function of the elapsed time after heating is started, and compared with both results measured using an

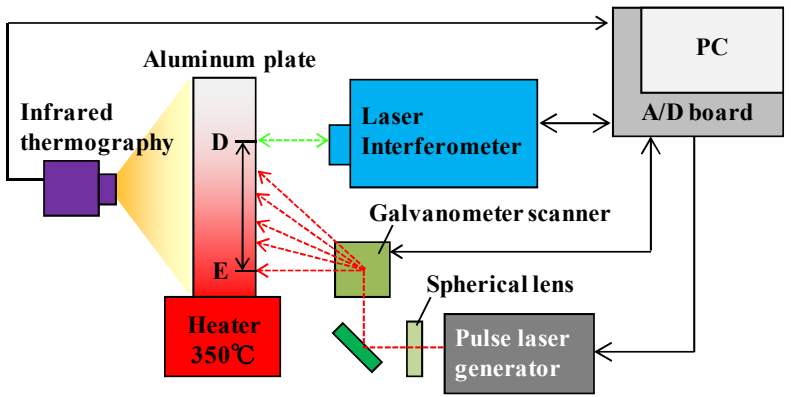

(a)

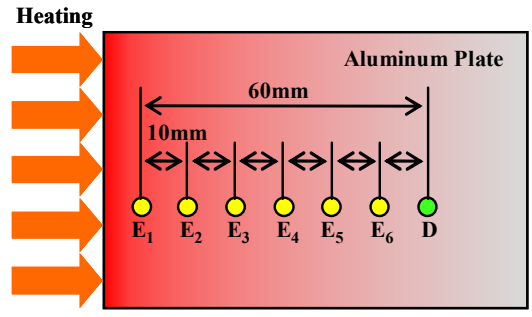

(b)

Fig. 2 Schematic of the experimental setup used. (a) Laser ultrasonic system with the pulsed laser scanning for measuring SAWs on a heated plate, (b) the pulsed laser irradiation points, $\mathrm{E}_{1}-\mathrm{E}_{6}$, and detection point $\mathrm{D}$. 
infrared camera and the former method [9] to verify the validity of the estimated results by the proposed ultrasonic method.

Figure 3 shows measured signals of SAW generated at positions from $E_{1}$ to $E_{6}$ on the aluminum surface before heating, where $\mathrm{SAW} \mathrm{E}_{i}$ means the wave generated at $\mathrm{E}_{i}$. As expected, the time delay of each SAW changes in proportion to the distance between the generated and detected positions. The center frequency of each wave is about $2 \mathrm{MHz}$. Although only slight decrease in the amplitude of the signal is observed, we can see that the pulsed laser scanning does not affect SAW generation significantly.

Figure 4 shows the change in the waveform of the SAW $E_{1}$ with the elapsed time after heating is started. It can clearly be seen that the echo is gradually delayed with the elapsed time because of rising of temperature of the aluminum. It is noted that the same tendency is observed for the other SAWs generated at different positions. The arrival time of SAW is determined by the zero-crossing method [11]. The variation in the time delay of each SAW with the elapsed time is determined by taking the cross-correlation of the measured signals of SAWs during heating [12].

Figure 5 shows the variations in the temperature and the time delay of the SAW $E_{1}$ during heating. As we expected, the temperature starts to rise immediately after the heating starts. Corresponding to this temperature rising, the time delay of the SAW $\mathrm{E}_{1}$ which is generated at a higher temperature region increases rapidly with the elapsed time. A small deviation in the time delay is due to the relatively low signal-to-noise ratio in the measured signals of the SAW. The similar results are obtained for other SAWs generated at $\mathrm{E}_{2}$ to $\mathrm{E}_{6}$. Using these time delays for SAW $\mathrm{E}_{i}(i=1$ to 6$)$, the surface temperatures at the middle point between the adjacent two positions, e.g. $E_{1}$ and $E_{2}, E_{2}$ and $E_{3}, E_{3}$ and $E_{4}$, etc., are determined from Eq. (4). The obtained five surface temperatures are combined together to construct the one-dimensional surface temperature distribution between $\mathrm{E}_{1}$ and $\mathrm{E}_{6}$. It is noted that the measurement and analysis processes for obtaining the temperature distribution are completed within $1 \mathrm{~s}$.

Prior to use the Eq. (4), it is necessary to obtain the relationship between the SAW velocity and temperature for the aluminum. The same procedure with the laser-ultrasonic system used in the previous work [9] is used for determining the temperature dependence of SAW velocity. The procedure is basically that a transit time of SAW is precisely measured for a certain distance of the aluminum surface as a function of temperature. The obtained temperature dependence of SAW velocity is shown in Fig. 6. It is found that the change in the SAW velocity is almost linear with temperature in the range from 25 to $200{ }^{\circ} \mathrm{C}$. Therefore, the temperature dependence is approximately given by

$$
v_{S A W}=-0.756 T+2981.7
$$

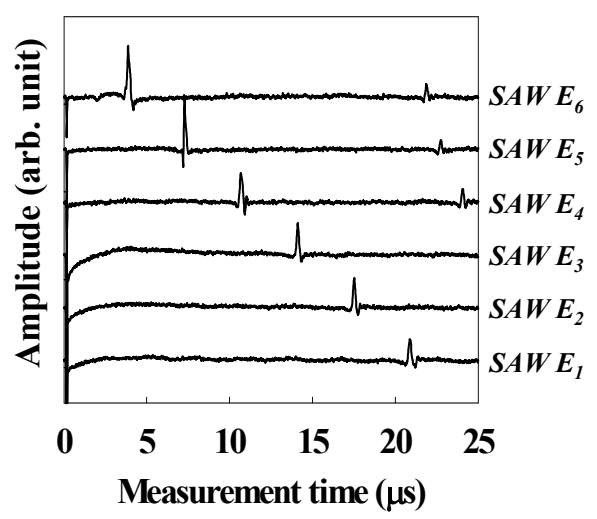

Fig. 3 Signals of SAWs generated at positions from $\mathrm{E}_{1}$ to $\mathrm{E}_{6}$ measured at room temperature.

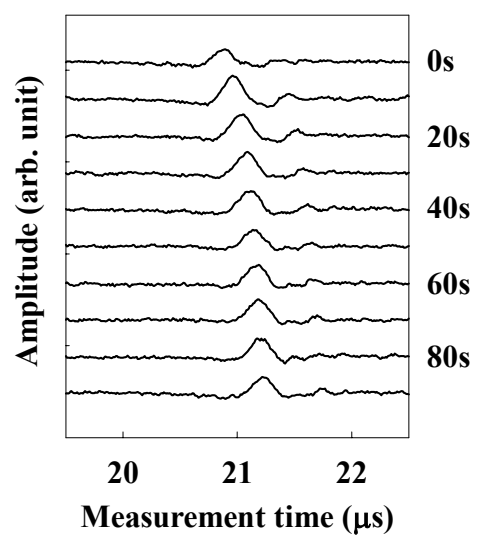

Fig. 4 Variations in waveforms of SAW $E_{1}$ during heating. 


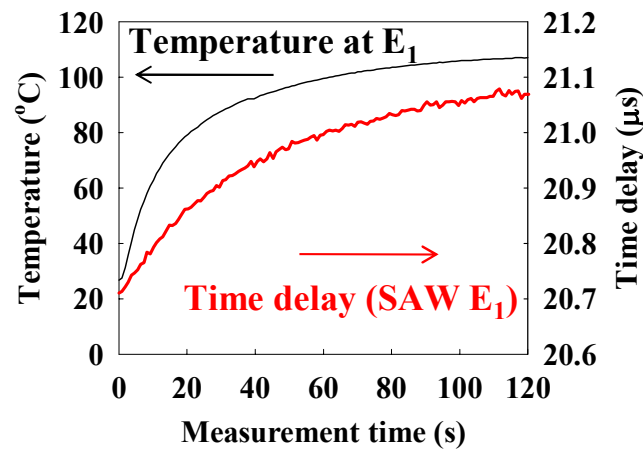

Fig. 5 Variations in the time delay of SAW $\mathrm{E}_{1}$ and the temperature at $\mathrm{E}_{1}$ on the plate during heating.

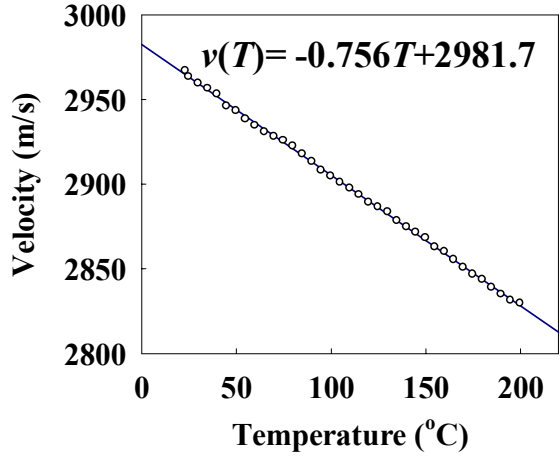

Fig. 6 Temperature dependence of the SAW velocity of the aluminum.

where the velocity is in $\mathrm{m} / \mathrm{s}$ and temperature $T$ is in degree Celsius. This relation is used in calculating surface temperatures.

Figure 7 shows the estimated surface temperature distributions for the aluminum plate and their variations with the elapsed time, where the estimated results by the proposed method are compared with those estimated by the former method [9] and measured using the infrared camera. Although there are small discrepancies among the results by the three methods, they almost agree with each other. It should be noted in Fig. 7 that the temperature at $60 \mathrm{~mm}$ from $E_{1}$ is used as a given (known) data in the former method. On the other hand, there is no need for such given data in the proposed method. No need for such given data becomes an advantage in practical use of the method. There exist large deviations at 25 and $45 \mathrm{~mm}$ in the estimated values by the proposed method. To clarify the reason and improve the measurement accuracy in the proposed method, further study is needed. In particular, it could be an important issue to examine the effect of the scan interval of pulsed laser irradiation on the accuracy.

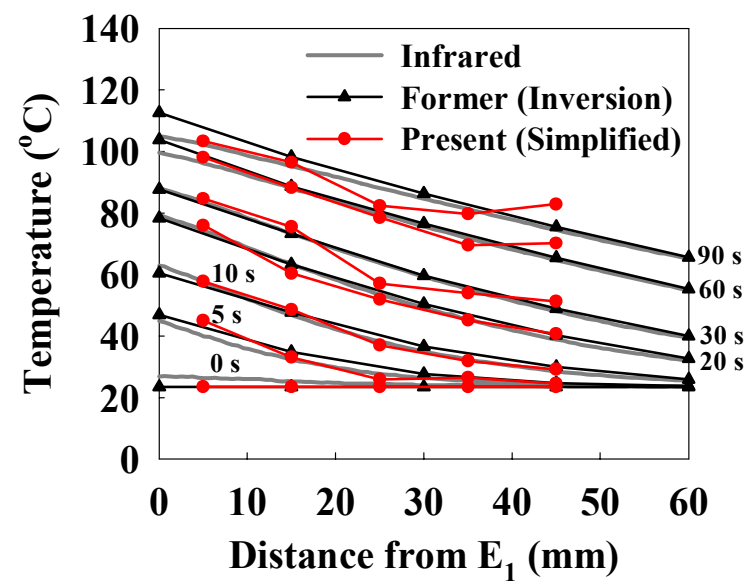

Fig. 7 Comparison among the surface temperature distributions measured by the present method, the former method and infrared radiation method.

\section{Conclusion}

A simple method with a laser ultrasound scanning for measuring surface temperature distributions on a material surface is presented. The feasibility of the proposed method is demonstrated through the experiment with aluminum plate heated up to $120^{\circ} \mathrm{C}$. It is noted that the method is applicable to any material as long as it has a flat surface. Although a 
further improvement in the measurement accuracy is necessary, it is expected that the method will be effective in the on-line monitoring of the transient variation in the surface temperature of the material processed at high temperatures. A foreseeable extension of this work would be to include quantitative evaluation of the accuracy of the proposed method in comparison with those of the other two methods. In addition, the influence of the curvature or roughness of material surface on the measurement accuracy should also be examined for providing practical applications of the proposed method.

\section{Acknowledgment}

The authors thank Mr. H. Yamada of Nagaoka University of technology for his support in this work. Financial support by the Grant-In-Aid for Scientific Research (B22360304) from the JSPS is greatly appreciated.

\section{References}

(1) Degertekin, F., L., Pei, J., Khuri-Yakub, B., T. and Saraswat, K., C., In situ acoustic temperature tomograghy of semiconductor wafers, Applied Physics Letters, Vol.64(1994), pp.1338-1340.

(2) Simon, C., VanBaren, P. and Ebbini, E., Two-dimensional temperature estimation using diagnostic ultrasound, Ultrasonics, Ferroelectrics, and Frequency Control, IEEE Transactions on, Vol.45(1998), pp.1088-1099.

(3) Chen, T.-F., Nguyen, K., T., Wen, S.-S., L. and Jen, C.-K., Temperature measurement of polymer extrusion by ultrasonic techniques, Measurement Science and Technology, Vol.10, No.3(1999), pp.139-145.

(4) Balasubramaniam, K., Shah, V., V., Costley, R., D., Boudreaux, G. and Singh, J., P., High temperature ultrasonic sensor for the simultaneous measurement of viscosity and temperature of melts, Review of Scientific Instruments, Vol.70(1999), pp.4618-4623.

(5) Huang, K., N., Huang, C., F., Li, Y., C. and Young, M., S., High precision, fast ultrasonic thermometer based on measurement of the speed of sound in air, Review of Scientific Instruments, Vol.73(2002), pp.4022-4027.

(6) Mizutani, K., Kawabe, S., Saito, I. and Masuyama, H., Measurement of temperature Distribution Using Acoustic Reflector Array, Japanese Journal of Applied Physics, Vol.45, No.5B (2006), pp.4516-4520.

(7) Takahashi, M. and Ihara, I., Ultrasonic Monitoring of Internal Temperature Distribution in a Heated Material, Japanese Journal of Applied Physics, Vol.47, No.5(2008), pp.3894-3898.

(8) Ihara, I. and Takahashi, M., Non-invasive Monitoring of Temperature Distribution inside Materials with Ultrasound Inversion Method, International Journal of Intelligent Systems Technologies and Applications, Vol.7, No.1(2009), pp. 80-91.

(9) Takahashi, M. and Ikuo, I., Quantitative Evaluation of One-Dimensional Temperature Distribution on Material Surface Using Surface Acoustic Wave, Japanese Journal of Applied Physics, Vol.48, No.7(2009), GB04-1.

(10) Pouet, B., Breugnot, S. and Clémenceau, P., Robust Laser-ultrasonic Interferometer Based on Random Quadrature Demodulation, AIP Conference Proceedings, Vol.820(2006), pp.233-239.

(11) Xu, Z., Carrion, L., and Maciejko, R., A zero-crossing detection method applied to Doppler OCT, Optics Express, Vol. 16, No. 7(2008), pp.4394-4412.

(12) Amamiya, Y., Singou Shori Nyumon (in Japanese), (1999), p.51, Ohm-sha. 